\title{
The period-radius relation of classical Cepheids and the problem of their mode identification
}

\author{
Mikhail Sachkov \\ Institute of Astronomy, Russian Academy of Sciences, Pyatnitskaya 48, 119017, Moscow, \\ Russia \\ email: msachkov@inasan.ru
}

\begin{abstract}
Based on radial-velocity measurements and photometric observations, we calculated the radii of around 200 classical Cepheids that were previously assumed to be fundamental-mode pulsators. Our detailed analysis of the period-radius diagram shows that the sample of Cepheids with pulsation periods shorter than 9 days probably contains a significant fraction (up to $30 \%$ ) of stars pulsating in the first overtone.
\end{abstract}

Keywords. stars: fundamental parameters, stars: pulsations, Cepheids 